\title{
Brownian Diffusion of Individual Janus Nanoparticles at Water/Oil Interfaces
}

\author{
Dapeng Wang, ${ }^{*}, \perp$ You-Liang Zhu, ${ }^{\perp}$ Yuehua Zhao, Christopher Y. Li, Ashis Mukhopadhyay, \\ Zhao-Yan Sun,* Kaloian Koynov,* and Hans-Jürgen Butt
}

Cite This: ACS Nano 2020, 14, 10095-10103

\section{ACCESS \\ Wlll Metrics \& More \\ Article Recommendations \\ Supporting Information}

ABSTRACT: Janus nanoparticles could exhibit a higher interfacial activity and adsorb stronger to fluid interfaces than homogeneous nanoparticles of similar sizes. However, little is known about the interfacial diffusion of Janus nanoparticles and how it compares to that of homogeneous ones. Here, we employed fluorescence correlation spectroscopy to study the lateral diffusion of ligand-grafted Janus nanoparticles adsorbed at water/oil interfaces. We found that the diffusion was significantly slower than that of homogeneous nanoparticles. We carried out dissipative particle dynamic simulations to study the mechanism of interfacial slowdown. Good agreement between experimental and simulation results has been obtained only provided that the flexibility of ligands grafted on the nanoparticle surface was taken into account. The polymeric ligands were deformed and oriented at an interface so that the effective radius of Janus nanoparticles is larger than the nominal one obtained by measuring the diffusion in bulk solution. These findings highlight further the critical importance of the ligands grafted on Janus nanoparticles for applications involving nanoparticle adsorption at an interface, such as oil recovery or two-dimensional self-assembly.

KEYWORDS: Brownian diffusion, Janus nanoparticles, fluorescence correlation spectroscopy, Stokes-Einstein equation, interfacial phenomena

$\mathrm{P}$ article adsorption at liquid interfaces is found ubiquitously in nature. ${ }^{1}$ The phenomenon is of considerable interest because it can be used to stabilize multiphase fluid mixtures and is important for various applications ranging from oil recovery to two-dimensional self-assembly. ${ }^{1,2}$ For many of these applications, the size and surface chemistry (ligand) of adsorbed particles are of key importance. If the two hemispheres of a particle surface are decorated with hydrophilic and hydrophobic ligands, i.e., in the form of Janus particles, adsorption at a water/oil interface allows both regions to be in contact with their preferred phases. $^{3-5}$ As a result, the adsorption of Janus particles is expected to be significantly stronger than that of particles with a chemically homogeneous surface, as predicted by theory ${ }^{6}$ and corroborated by experiments and simulations. ${ }^{7-13}$

Many applications rely not only on the ability of particles to adsorb on an interface but also on their interfacial mobility after adsorption, which, however, often exhibits anomalies and is not yet fully understood. For example, it was shown that such particles took untenably long to reach equilibrium after adsorption to a liquid interface. ${ }^{14,15}$ Furthermore, the translational and rotational diffusions of adsorbed particles were often found to be slower than the predictions of the Stokes-Einstein relation and hydrodynamic theories, ${ }^{16-25}$ whereas the underlying mechanisms were not captured by the simulations. ${ }^{26,27}$ Some reasons for the slow diffusion of micron-sized particles have been suggested such as the ubiquitous surface "defects" that induce multiple metastable pinning of the three-phase contact line, ${ }^{28-30}$ producing an extra reverse random force to slow diffusion. ${ }^{19}$ However, according to the Young-Laplace equation, contact line deformation should be vanishingly small when the particle size is $\sim 10 \mathrm{~nm}^{31}$ Therefore, whether the contact line deformation mechanism works for nanoparticles remains unknown. This scenario may be even more complicated for amphiphilic Janus nanoparticles (JNPs) because the boundary between the chemically distinct regions

Received: April 20, 2020

Accepted: July 14, 2020

Published: July 14, 2020 

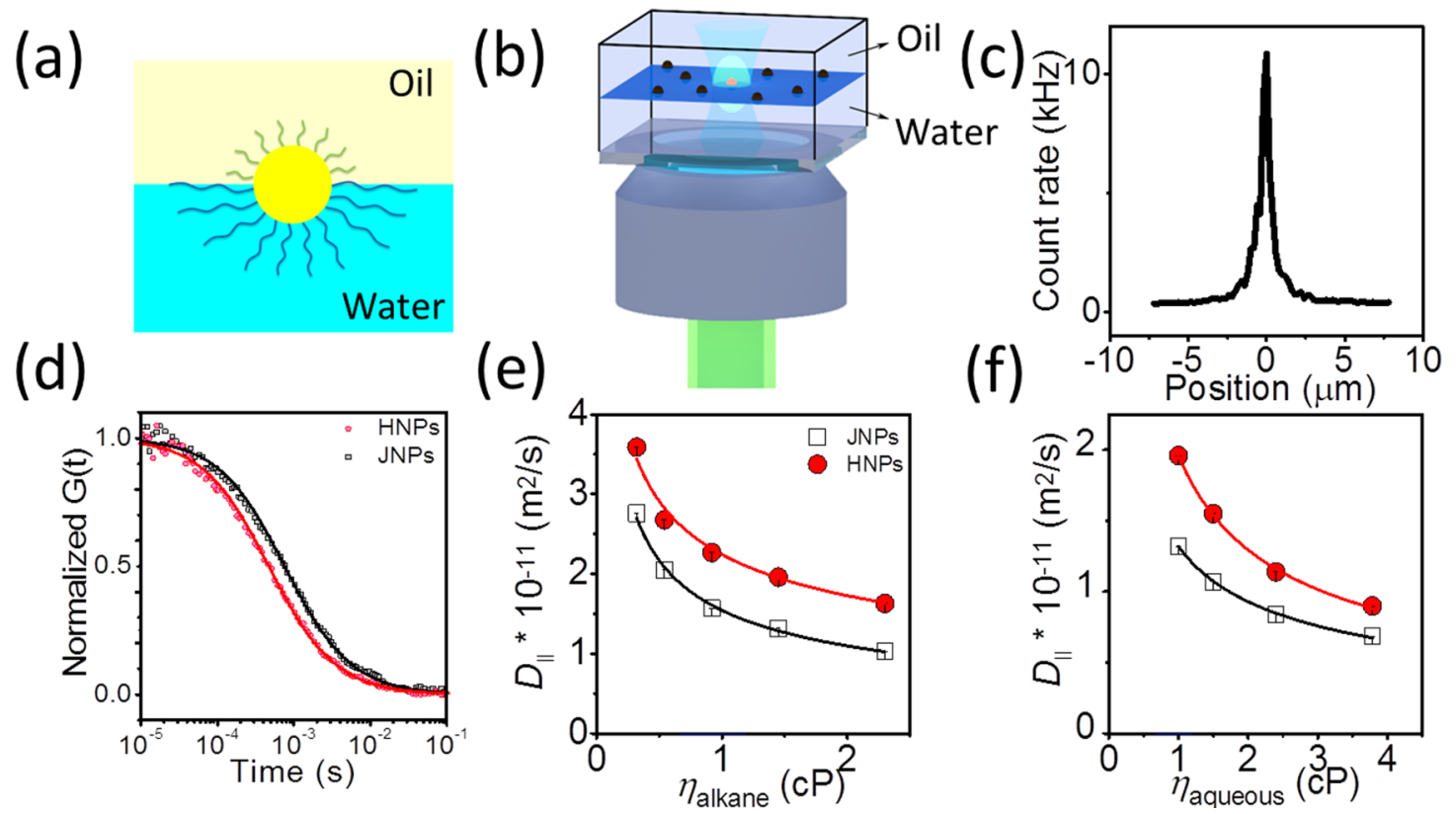

Figure 1. (a) Schematic representation of ligand-grafted JNPs at a water/oil interface. The yellow sphere and green and blue strings denote the quantum dot core, 1-dodecanethiol, and PEG, respectively. (b) Schematic representation of the FCS experiments at the water/oil interface. (c) Fluorescence intensity scan through the water/decane interface with adsorbed JNPs. (d) Representative autocorrelation curves (symbols) and their fits with eq 1 (solid lines) for JNPs (black squares) and HNPs (red circles) at water/decane interfaces. $D_{\text {exp,ll }} v e r s u s$ viscosity of the (e) alkane and (f) aqueous phases at interfaces with adsorbed JNPs (circles) and HNPs (squares). The viscosity of the aqueous phase was controlled by mixing water with glycerol.

is expected to be an intrinsic defect, supposedly causing strong pinning. Thus, precise experimental data directly comparing the interfacial diffusion of small amphiphilic JNPs to that of homogeneous nanoparticles (HNPs) of similar sizes are needed in order to properly understand the underlying physical phenomena.

In this work, we studied the diffusion of small $(\sim 10 \mathrm{~nm})$ JNPs and HNPs at water/alkane interfaces as a function of the viscosity of the aqueous and the alkane phases. We found that the two-dimensional diffusion of JNPs at water/oil interfaces was slower compared to that of HNPs and to the predictions of a modified Stokes-Einstein equation using the friction coefficient of spherical particles. We performed dissipative particle dynamic simulation to explore the mechanisms of interfacial slowdown and found out that it can be attributed to the orientation of ligands on a nanoparticle surface.

\section{RESULTS AND DISCUSSION}

Enabling the Quantification of the Individual JNPs' Diffusion at a Water/Oil Interface. The JNPs were synthesized through a method combining "solid-state grafting-to" and "grafting-from" using CdSe quantum dots with a mean radius of about $3.6 \mathrm{~nm}$ as seeds. ${ }^{32}$ Thiol-terminated poly(ethylene glycol) (PEG, molecular weight $\left[M_{\mathrm{w}}\right] 5 \mathrm{~kg} / \mathrm{mol}$ ) single crystals were used as a solid substrate that was able to adsorb the native quantum dots. The side that had contact with the substrate was grafted with PEG via the ligand exchange reaction to irreversibly displace the native oleate ligands on the quantum dot surface. The oleate ligands on the other side were exchanged with 1-dodecanethiol. After dissolving the single-crystal substrates, JNPs were obtained. HNPs were prepared by functionalizing the same quantum dots with thiol-terminated PEG on the entire surface. Because JNPs can be dispersed only in dichloromethane, we determined the hydrodynamic radius $\left(R_{\mathrm{H}}\right)$ by measuring diffusion in a dilute dichloromethane dispersion; the resulting $R_{\mathrm{H}}$ 's of JNPs and HNPs are 5.0 and $6.4 \mathrm{~nm}$, respectively, as shown in Figure S6 in the Supporting Information.

JNPs dissolve neither in an aqueous medium nor in alkane. Therefore, we cannot disperse them into one phase and wait for them to adsorb to the interface spontaneously. In order to circumvent this difficulty, a small amount (about 4-10 $\mu \mathrm{L}$ ) of JNPs' dichloromethane dispersion at a concentration of $10^{-10}$ $\mathrm{M}$ was gently placed upon a water surface using an Eppendorf adjustable-volume $(0.5-10 \mu \mathrm{L})$ pipet. Although the density of dichloromethane $\left(1.33 \mathrm{~g} / \mathrm{cm}^{3}\right.$ at $\left.25{ }^{\circ} \mathrm{C}\right)$ is greater than that of water $\left(1.00 \mathrm{~g} / \mathrm{cm}^{3}\right.$ at $\left.25{ }^{\circ} \mathrm{C}\right)$, the dichloromethane would spread at the water/air interface provided that the volume is sufficiently small because of the high interfacial tension between water and air $\left(71.99 \mathrm{mN} / \mathrm{m}\right.$ at $\left.25{ }^{\circ} \mathrm{C}\right)$. The dichloromethane solvent was allowed to evaporate for typically $15 \mathrm{~s}$. Afterward, the alkane phase was added carefully on top of the water surface. The resulting surface coverage of the NPs was around $1-2 \mu \mathrm{m}^{2}$ per particle.

Although a large number of experimental techniques can be used to study translational diffusion, very few can measure precisely the interfacial diffusion with a high spatial/temporal resolution. Here we chose fluorescence correlation spectroscopy (FCS) that is very well suited for such a task. ${ }^{33-35}$ This is because the concept of FCS is based on monitoring the fluorescence fluctuations originating from fluorescent species diffusing in and out of a very small $\left(<1 \mu \mathrm{m}^{3}\right)$ confocal volume (Figure 1b). In a representative experiment, the confocal volume was first scanned across the water/alkane interface with adsorbed nanoparticles to find its precise location at the position of the maximum detected fluorescence (Figure 1c). The confocal volume was then positioned at the interface, and the fluctuations in detected fluorescence intensity $F(t)$ caused 
by the lateral diffusion of the nanoparticles were measured and used to compute an experimental autocorrelation function, $G(\tau)=1+\langle\delta \mathrm{F}(t) \delta F(t+\tau)\rangle /\langle F(t)\rangle^{2}$ where $\delta F(t)=F(t)-$ $\langle F(t)\rangle$. Representative autocorrelation functions of JNPs and HNPs at a water/decane interface are illustrated in Figure 1d. Finally, the experimental autocorrelation function was fitted with the analytical equation ${ }^{36,37}$

$$
G(\tau)=1+\frac{1}{N} \frac{1}{\left[1+\frac{\tau}{\tau_{\mathrm{D}}}\right]}
$$

to obtain the average number of diffusing species $N$ and their mean diffusion time $\left(\tau_{\mathrm{D}}\right)$, which is directly related to the twodimensional diffusion coefficient via $D_{\text {exp, }}=r_{0}{ }^{2} / 4 \tau_{\mathrm{D}}$, where $r_{0}$ is the radial dimension of the confocal volume. Here $r_{0}=220$ $\mathrm{nm}$ is the lateral dimension of the confocal detection volume. This value was obtained by measuring the diffusion time of rhodamine $6 \mathrm{G}$ molecules with a known hydrodynamic radius in a dilute aqueous solution.

Interfacial Diffusion Measured by FCS. For a spherical particle straddling a liquid-liquid interface, the viscous drag can be approximated according to the particle's cross-sectional area immersed by the respective fluid phases using a generalized Stokes-Einstein relation: ${ }^{38,39} \quad D_{\mathrm{SE}, \|}=2 \pi k_{\mathrm{b}} T$ / $\left(6 \pi R_{\mathrm{H}}\left(2 \pi \eta_{\mathrm{w}}+\left(\eta_{\mathrm{a}}-\eta_{\mathrm{w}}\right)(2 \Theta-\sin 2 \Theta)\right)\right)$. Here, $D_{\mathrm{SE}, \|}$ is the theoretical diffusion coefficient; $\Theta, \eta_{w}$, and $\eta_{a}$, are the contact angle and the viscosities of the aqueous and alkane phases, respectively. It is difficult to determine $\Theta$ of nanoparticles. $^{29,30,40,41}$ Therefore, we first measured the diffusion at a water/decane interface because the viscosities of water (1 $\mathrm{cP})$ and decane $(0.92 \mathrm{cP})$ at $20^{\circ} \mathrm{C}$ are almost identical. In this scenario, the diffusion coefficient does not depend on $\Theta$ and can be approximated using the classic Stokes-Einstein form $D_{\mathrm{SE}, \|} \approx k_{\mathrm{b}} T / 6 \pi R_{\mathrm{H}} \eta_{\mathrm{w}}$. Figure $1 \mathrm{~d}$ shows representative autocorrelation curves (ACCs) and their fits for diffusion of JNPs and HNPs at a water/decane interface. We found a noticeably higher average diffusion time through the FCS observation volume for JNPs than for HNPs, indicating that the Janus character plays a role in interfacial diffusion. Furthermore, the experimental values of the diffusion

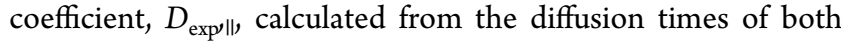
nanoparticles were much smaller than $D_{\mathrm{SE}, \mathrm{ll}}: D_{\text {exp, },} / D_{\mathrm{SE}, \mathrm{ll}} \approx 0.35$ for JNPs and $D_{\text {exp,\|l }} / D_{\mathrm{SE}, \|} \approx 0.77$ for HNPs.

We further measured $D_{\text {exp, }}$ of both nanoparticles versus the viscosities of the aqueous and alkane phases (Figure 1e,f). We found that the interfacial diffusion coefficient decreases with increasing the viscosity of both aqueous and alkane phases, indicating that the particles are partially immersed in both phases. It is noteworthy that the interfacial diffusion of JNPs is slower than that of HNPs at all conditions.

It is important to consider and exclude potential fallibilities that could affect the accuracy of the experimental results. For example, the presence of nanoparticle aggregates, which are larger than individual nanoparticles, could lead to a longer diffusion time. This possibility was excluded because aggregates are easily recognized in FCS experiments as anomalously bright spikes in the intensity-time trace. Another possible source of error could be desorption from the interface ${ }^{42-44}$ because the FCS technique relies on detection of diffusion into and out of a confocal volume shaped like an erect ellipse (Figure 1b). Under such circumstances, desorbed NPs could potentially take longer than the laterally diffusing ones to exit the confocal volume, producing a false appearance of slow diffusion. Although we cannot exclude this possibility for HNPs, this scenario is not possible for JNPs that are not dispersible either in water or in alkanes. Finally, we have ruled out the possibility of contamination as a source of the slowdown, because the addition of several types of surfactants, including sodium dodecyl sulfate, cetrimonium bromide, and tetraethylene glycol monooctyl ether $\left(\mathrm{C}_{8} \mathrm{E}_{4}\right)$, at a concentration of $10^{-5} \mathrm{M}$ in the aqueous solution did not cause a decrease in $D_{\text {exp,\| }}$ within the experimental uncertainty. Thus, after a series of control experiments, we concluded that the experimentally observed slowdown in the interfacial diffusion is not originated from artifacts.

Stepwise Analysis toward the Mechanism of Interfacial Slowdown by Using the Dissipative Particle Dynamics (DPD) Simulation. Next, we attempt to interpret the observed diffusional slowdown of the studied nanoparticles at water/oil interfaces. We note two pioneering theories that describe well the interfacial diffusion slowdown for homogeneous microparticles. One of these theories considers the hydrodynamic interactions, ${ }^{39,45-51}$ and the other takes the contact line fluctuation into account. ${ }^{19,21}$ However, because of the JNPs' surface heterogeneity, it is difficult to analytically describe the interfacial diffusion of JNPs. To overcome this difficulty, the DPD simulation ${ }^{52}$ was selected, after screening many other simulation methods, because of its suitability to involve the hydrodynamic interaction, relatively low computational loading, and our ability to enable the large-scale parallel computing for this method. ${ }^{52}$ These advantages allowed us to rapidly test the diffusion of a series of simulated model nanoparticles at an interface between immiscible liquids, to explore the dominant mechanism in the interfacial diffusion of JNPs, and to understand why it is even slower than that of HNPs.

The DPD simulations were carried out in NVT ensembles. The simulation time of a single trajectory is typically $10^{7} \tau$, with a time step $\Delta t=0.02 \tau$ where $\tau=6.9$ ps. The simulation method is described in the Methods section. A full version is given in the Supporting Information. The simulation setting was calibrated by measuring the diffusion of a smooth nanoparticle in bulk media. This simulated result is consistent with the prediction of the Stokes-Einstein equation, justifying the fitness of the simulation parameters.

We examined a series of simulated model nanoparticles to make a stepwise determination in exploring the key factor that is responsible for the measured phenomena. The diameters of the rigid core of all simulated nanoparticles were set to be 5 (corresponding to $5 \mathrm{~nm}$ in real units). For these simulated model nanoparticles, we calculated the ensemble-averaged translational diffusion coefficient either in dilute dispersion $\left(D_{\mathrm{DPD}, \text { bulk }}\right)$ or at interfaces $\left(D_{\mathrm{DPD}, \mathrm{Il}}\right)$ via $D_{\mathrm{DPD}}=\mathrm{MSD} / 4 \Delta t$, where MSD denotes the mean squared displacement.

We used a single-site model to produce a smooth nanoparticle by expanding a DPD bead. The size was tuned by a parameter $d$; herein, $d=1$ for solvent bead and $d=5$ for the expanded nanoparticle. DPD forces have three terms, namely, the conservative force, random force, and dissipative force, respectively, as explained in the Methods section. The conservative force formula for the smooth nanoparticle system is rewritten as 

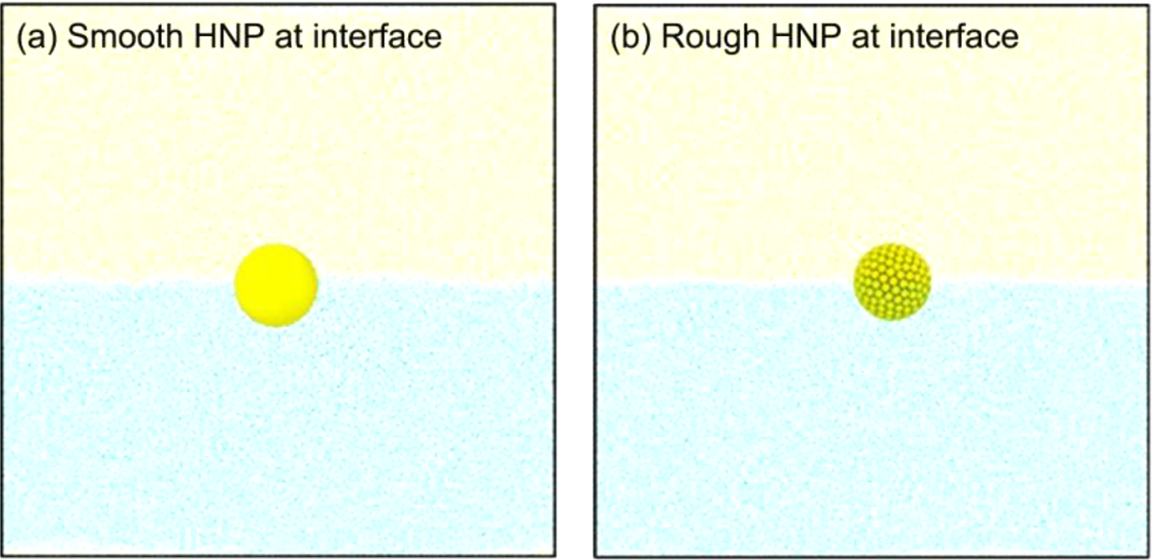

(c) Rough JNP at interface
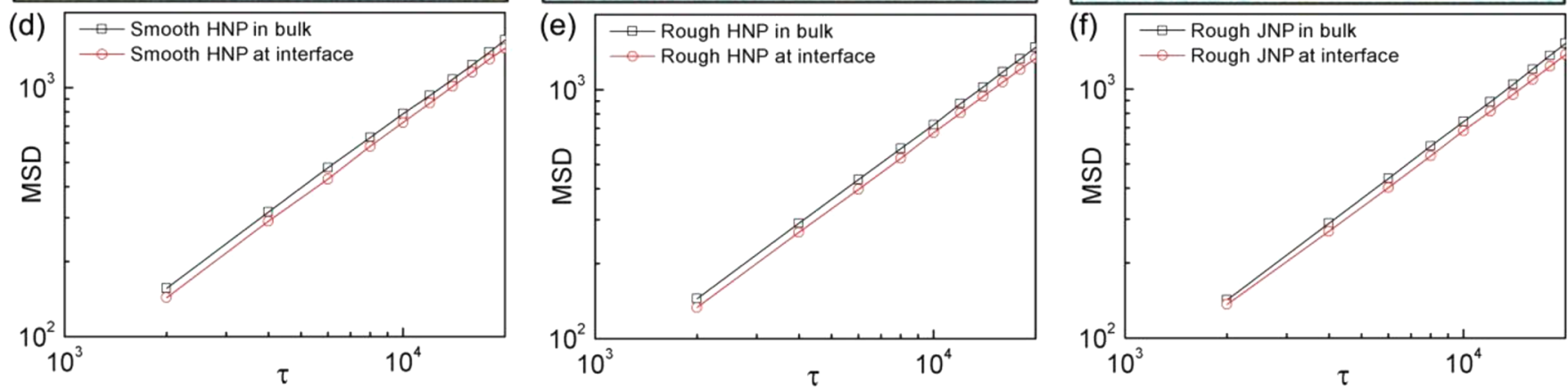

Figure 2. Snapshots of (a) a smooth nanoparticle, (b) a homogeneous nanoparticle that has a rough surface, (c) a Janus nanoparticle that has a rough surface. Mean squared displacement (MSD) of (d) a smooth nanoparticle, (e) a homogeneous nanoparticle that has a rough surface, (f) a Janus nanoparticle that has a rough surface in bulk dispersion and at anterface.

$$
\begin{aligned}
\vec{F}_{i j}^{\mathrm{C}}= & -\nabla V\left(r_{i j}-\Delta\right) \\
= & \left\{\begin{array}{cc}
\alpha_{i j}\left(1-\frac{r_{i j}-\Delta}{r_{c}}\right) \vec{e}_{i j}, & r_{i j} \leq r_{\mathrm{c}}+\Delta \text { and } r_{i j} \geq \Delta \\
\alpha_{i j} \overrightarrow{i j}_{i j}, & r_{i j}<\Delta \\
0, & r_{i j}>r_{\mathrm{c}}
\end{array}\right.
\end{aligned}
$$

where $\alpha$ is an additional parameter and $\Delta=d_{i}+d_{j}-1$ represents the size of two beads with diameter $d_{\mathrm{i}}$ and $d_{\mathrm{j}}$, respectively. Other parameters are explained in the Methods section. For two solvent beads with $d_{i}=d_{i}=1, \Delta=0$; the conservative force returns to normal expression.

We simulated the diffusion of a smooth NP at a liquid/liquid interface and compared it to bulk diffusion, as shown in Figure 2a. The purpose of studying a smooth NP is to eliminate the effect of contact line pinning. The resulting $D_{\mathrm{DPD}, \mathrm{Il}} / \mathrm{D}_{\mathrm{DPD}, \mathrm{bulk}}$ is equal to 0.94; this result is irrespective of the surface chemistry. We hypothesized that the small difference is caused by hydrodynamic interaction. To quantify this effect, we placed a smooth nanoparticle in a position close to the liquid/liquid interface and quantified the distance-dependent diffusion coefficient to the interface. We found a gradual decrease in $D_{\mathrm{DPD}, \|}$ as a function of proximity to the interface (Figure S4 in the Supporting Information); the diffusion coefficient in a position close to the interface is comparable to $D_{\mathrm{DPD}, \|}$, which is consistent with a scenario of hydrodynamic coupling between a spherical particle and a planar liquid/liquid interface. ${ }^{50}$ The hydrodynamic coupling may play a role in interface diffusion of HNPs, but cannot interpret the diffusional behavior of JNPs.
We further explored the effect of contact line pinning on the diffusion. The adsorption of nanoparticles at a liquid/liquid interface may cause local interfacial deformation at the triple line. Such deformation may be associated with the interfacial capillary waves, particle wettability, and particle size/ weight. $^{19,53}$ The wetting-driven interfacial deformations may drive the system to deviate from equilibrium. Thus far, the scenario involving contact line pinning of JNPs and its possible influence on the diffusion remain elusive. Thus, we exploit the DPD simulation to explore this effect in detail with appropriate nanoparticle models. Because the contact line pinning can be induced by surface defects, ${ }^{54}$ we constructed an HNP (Figure 2b) and a JNP (Figure 2c) that have a rough surface by stacking a bundle of DPD beads into a sphere. The integration of the sphere for motion and rotation is achieved by a rigid body method. If the DPD beads of different hemispheres were assigned to different values of $\alpha_{i j}$ (the parameter to determine the magnitude of the repulsive force between particles $i$ and $j$ ), a JNP takes shape, as shown in Figure 2c. If $\alpha_{i j}$ of all DPD beads of the sphere is identical, the sphere is an HNP (Figure $2 \mathrm{~b})$. The presence of topographic caves could act as a free energy metastable minimum to transiently lock the three-phase contact line. ${ }^{27}$ Using such nanoparticle models, we successfully observed contact line pinning in a simulation run (Figure S5 in the Supporting Information), but this phenomenon did not cause a noticeable decrease in the diffusion coefficient. The resulting $D_{\mathrm{DPD}, \mathrm{Il}} / D_{\mathrm{DPD} \text {,bulk }}$ of JNPs and HNPs was 0.91 and 0.92 , as fit by the MSD data shown in Figure 2e. These results are consistent with recent simulation results, ${ }^{27}$ indicating that contact line fluctuation is likely not the key mechanism for the slowing down of JNPs at interfaces. 
The failure of the aforementioned two models signified that these models overlooked a key feature causing an anomalous increase of the drag coefficient. Previous studies showed that the flexible polymer ligands grafted on a nanoparticle can control the nanoparticles' properties at a water/oil interface. $^{43,55-62}$ Inspired by these studies, we hypothesized that PEG deformation and reorientation on a JNP make the effective radius at an interface larger than the nominal one measured in bulk diffusion. Comparatively, PEG ligands on an HNP were expected to rearrange asymmetrically, because PEG collapses in oil. ${ }^{57}$ To test this hypothesis, we designed ligandgrafted model JNPs and HNPs. A group of DPD beads, denoted as type $G$, was stacked to form a sphere. A rigid body method was used to make the sphere a rigid object. We modeled the grafted PEG by a hybrid scheme involving two types of DPD beads, denoted as $A$ and $B$, respectively. The group $B$, comprising three carbon and two oxygen atoms, is more hydrophilic than group $A$, which included four carbon atoms and one oxygen heavy atom (see Figure $S 1$ in the Supporting Information for details). A $5 \mathrm{~kg} / \mathrm{mol} \mathrm{PEG} \mathrm{chain}$ was modeled as a hybrid bead string that consisted of 42 beads: $28 A$ and $14 \mathrm{~B}$. The 1-dodecane was modeled as a string of three $A$ beads. To model a ligand-grafted Janus nanoparticle, 60 PEG chains were grafted on one hemisphere and 601 dodecane chains on the other. To model a ligand-grafted homogeneous nanoparticle, 120 PEG chains were grafted uniformly on the nanoparticle surface. The beads were connected via harmonic springs that are described in the Methods section with $k=4.0$ and $r_{0}=0$. The core of a ligandgrafted nanoparticle consisted of 192 beads to make the nanoparticle density comparable to that of the solvent matrix. The cutoff distance $r_{c}$ is 1 . The pairwise conserve force parameters $\alpha$ are given in Table S2 in the Supporting Information.

Using this model, the simulation showed collapse of the PEG chain on the JNP and HNP surface in bulk solution (Figure 3a,c), mimicking the behavior of PEG in dichloromethane. After a JNP was adsorbed at a liquid/liquid interface, most of the PEG ligands were swollen and oriented parallel to the interface, and only a few remained in the bulk phase (Figure 3b). This is further demonstrated in Figure S7, which shows the one-dimensional radial density profile of a JNP in simulated bulk "water" and at a liquid/liquid interface. We found that the density profile in bulk water decays more rapidly than that at an interface, corroborating polymer swelling when a ligand-grafted JNP was adsorbed at a liquid/ liquid interface. Next, we quantified the normalized onedimensional PEG density profiles in the $x$ - and $z$-directions and two-dimensional PEG density mapping of an adsorbed JNP and HNP at a liquid/liquid interface, as shown in Figure $3 \mathrm{e}-\mathrm{h}$. The mapping of the adsorbed JNP and HNP was taken along the transversal section of the Janus boundary and the threephase contact line, respectively.

We begin with the analysis of the one-dimensional PEG density profiles. For a JNP, the density profile in the $z$ direction is nearly symmetric and decays rapidly with the distance from the interface, indicating that most of PEG chains are enriched at the interfacial boundary. However, for HNPs, the density profile in the $z$-direction is asymmetric; it decays much more slowly than that found for JNPs in the simulated "water" phase, indicating that many PEG chains were still dispersed in the bulk "water" phase. The interfacial enrichment of grafted PEG on a JNP's surface relative to an HNP's surface (a)

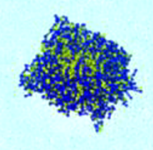

(b)

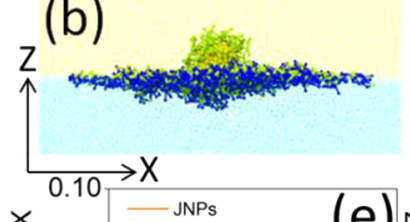

(d)
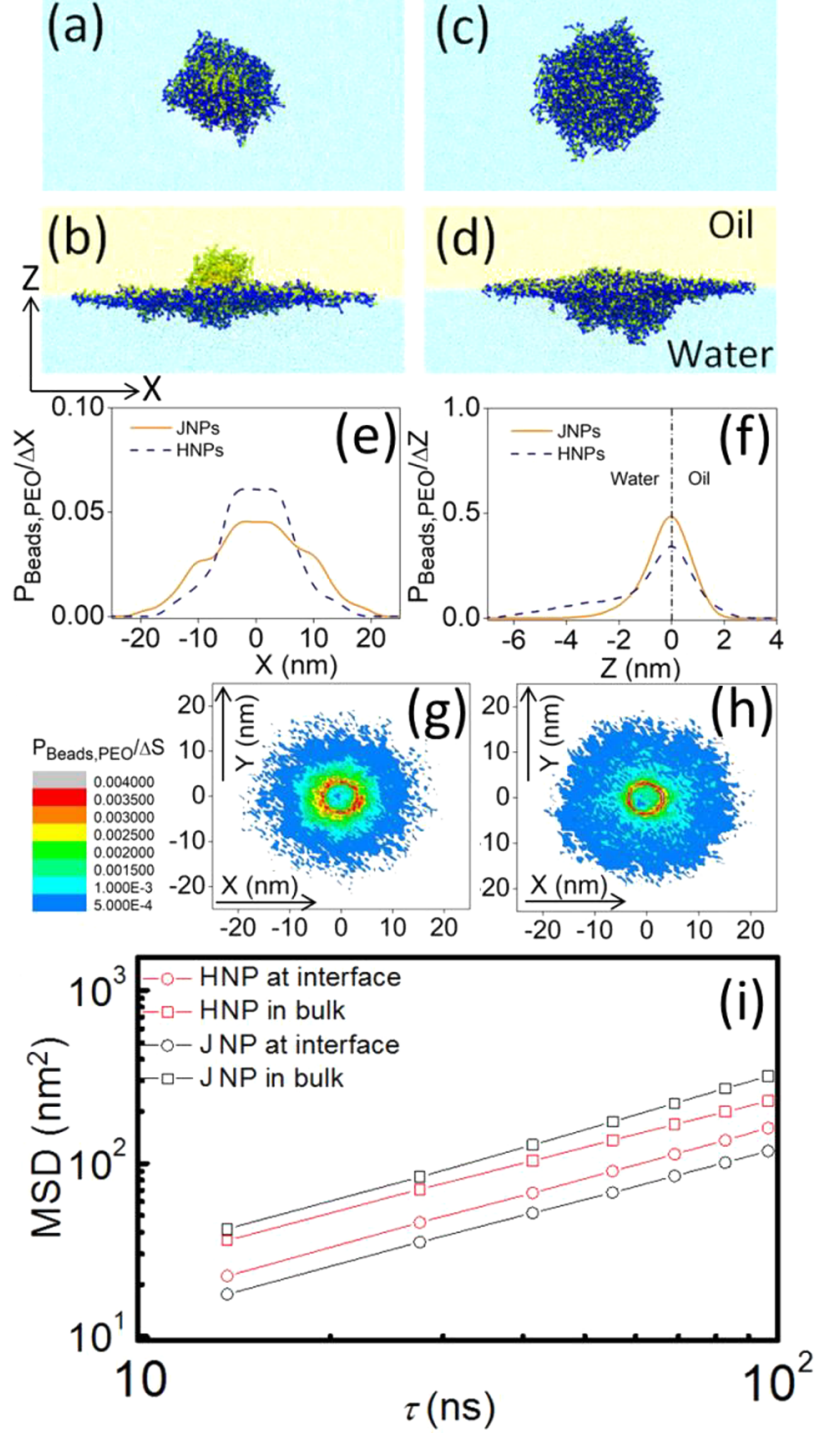

Figure 3. Snapshot of a ligand-grafted JNP (a) in bulk and (b) at an interface. Snapshot of a ligand-grafted HNP (c) in bulk and (d) at an interface. A coordinate near panel (b) denotes the $x$ - and $z$ directions. Normalized one-dimensional PEG density profiles of adsorbed HNP and JNP at a liquid/liquid interface in (e) $x$ - and (f) $z$-directions. Two-dimensional PEG density mapping of (g) adsorbed HNP and (h) JNP at a liquid/liquid interface. The mapping of the adsorbed JNP and HNP was taken along the transversal section of the Janus boundary and the three-phase contact line, respectively. (i) Mean squared displacement of JNPs and HNPs in bulk dispersion and at anterface.

(Figure 3d) causes a slower decay of the PEG density profile in the $x$-direction (Figure $3 \mathrm{e}$ ) and a more extended interfacial occupation (Figure $3 \mathrm{~g}, \mathrm{~h}$ ). The enrichment may be attributed to the decreased steric interaction at the Janus boundary because only short ligands were grafted onto the other half of the particle.

Next, we calculated the MSDs and the diffusion coefficients of JNPs and HNPs in bulk dispersion and at an interface as shown in Figure $3 \mathrm{i}$. The resulting $D_{\mathrm{DPD}, \mathrm{ll}} / \mathrm{D}_{\mathrm{DPD}, \text { bulk }} \approx 0.72$ for HNPs and $D_{\mathrm{DPD}, \mathrm{ll}} / D_{\mathrm{DPD} \text {,bulk }} \approx 0.35$ for JNPs were in good agreement with the experimental FCS observations.

Taken together, these results confirm the hypothesis that PEG deformation and reorientation on the nanoparticles is the 
reason for the slower interfacial diffusion. Indeed, we found that on the HNP surface the PEG ligands are swollen only near the interface. The deformation of PEG ligands makes the nanoparticle adopt a stretched oblate shape, consistent with previous simulation results involving ligand-grafted and hydrogel-made nanoparticles at liquid/liquid interfaces. ${ }^{63-65}$ This causes overall a small increase in the effective radius (decrease in the diffusion coefficient) when an HNP moves from bulk to an interface. On other hand, for a JNP all surface ligands are swollen when it is adsorbed at an interface, resulting in a noticeable increase in the effective radius and thus slower interfacial diffusion.

To highlight further the significance of polymer conformation, we studied the effect of the relative sizes of the hard-core and the soft ligands on the interfacial diffusion. In doing so, we took ligand-grafted model JNPs as shown in Figure 3a,b; the length of hydrophobic ligands is deliberately set to be the same as that of PEG ligands on the other hemisphere. Using these model nanoparticles, we performed DPD simulation and calculated the $D_{\mathrm{DPD}, \mathrm{II}} D_{\mathrm{DPD}, \text { bulk }}$ of JNPs and HNPs as a function of the PEG length. Due to the limitation of computational capability, the longest length of grafted PEG that we simulated was $15 \mathrm{~kg} / \mathrm{mol}$. We found that $D_{\mathrm{DPD}, \mathrm{II}} D_{\mathrm{DPD} \text {, bulk }}$ of both JNPs and HNPs decreases monotonously with increasing the PEG length (Figure S9). Notability, values of $D_{\mathrm{DPD}, \| /} D_{\mathrm{DPD}, \text { bulk }}$ of JNPs are much smaller than that of HNPs over the studied range of PEG length. These results confirm that the surface ligands play a dominant role in the interfacial diffusion.

Variation of the Interfacial Tension Influences the Interfacial Diffusion. Despite having achieved excellent simulation-experiment consistency by taking the ligand flexibility into account, we sought to confirm that this model correctly captured the underlying mechanism rather than a phenomenological coincidence. The deformation of the polymer shell around the inorganic core of a nanoparticle is expected to be stronger at a water/oil interface of higher interfacial tension. This is because, at such an interface, the energy gain of extending the polymer chains is greater since more surface area can be covered. ${ }^{6}$ Therefore, decreasing the interfacial tension is expected to result in faster diffusion of ligand-grafted nanoparticles. This simple argument was confirmed by both experiments and simulations.

We first studied whether the interfacial tension can influence the surface activity of polymeric ligands by comparing the adsorption isotherm at water/octane and water/toluene interfaces, respectively. The viscosities of octane $(0.54 \mathrm{cP})$ and toluene $(0.59 \mathrm{cP})$ at $20{ }^{\circ} \mathrm{C}$ are comparable, but the interfacial tension of a water/octane interface $(52 \mathrm{mN} / \mathrm{m}) \mathrm{far}$ exceeds that of a water/toluene interface $(36 \mathrm{mN} / \mathrm{m})$. Therefore, we can isolate the effect of interfacial tension by directly comparing the behavior at these two interfaces. The surface excess $\Gamma$ of the adsorbed component (PEG) can be calculated using the Gibbs equation:

$$
\Gamma=-\left.\frac{a}{R T} \frac{\partial \gamma}{\partial a}\right|_{T}
$$

Here, $R, T, a$, and $\gamma$ are the molar gas constant, temperature, interfacial activity, and interfacial tension, respectively. Equation 3 shows that $\Gamma$ is closely associated with $\partial \gamma / \partial a$ that is determined by the surface activity of individual adsorbents and the number of adsorbents in the dilute limit. As a rough approximation, we can replace the activity $a$ with the surface concentration $c$ with $\partial \gamma / \partial a \approx \Delta \gamma / \Delta c$. Therefore, to describe $\Gamma$ of individual adsorbents, one could measure the gradient of the adsorption isotherm for $c \rightarrow 0$.

Figure S8 shows experimental data of equilibrated interfacial tension versus the bulk PEG concentration for $c \rightarrow 0$ at different interfaces. PEG can indeed lower the interfacial tension more efficiently at a water/octane interface than at a water/toluene interface. Applying a linear fit to the $\gamma-c$ data, we found that values of $\Delta \gamma / \Delta c$ are equal to $1.3 \times 10^{7}$ and $4.3 \times$ $10^{6} \mathrm{Nm}^{-1} \mathrm{M}^{-1}$ at water/octane and water/toluene interfaces, respectively. As expected, the value of $\Delta \gamma / \Delta c$ obtained in the dilute limit is greater at a water/octane interface than at a water/toluene, evidently indicating that the interfacial tension could influence the surface excess of individual PEG chains. On one hand, these measurements verified that varying the interfacial tension can cause a change in the PEG conformation; on the other hand, the $\gamma$-dependent polymer conformational change can provide a means to examine whether the concept of polymer deformation is responsible for the interfacial slowdown.

Next, we performed FCS experiments to compare the diffusion of JNPs at water/octane and water/toluene interfaces. We found that the variation of interfacial tension did influence diffusion. ACCs at a water/toluene interface decay more rapidly than at a water/octane interface, reflecting an increase of $D_{\|}$from $2.3 \times 10^{-11}$ to $3.6 \times 10^{-11} \mathrm{~m}^{2} / \mathrm{s}$ (Figure $4 \mathrm{a}$ ). The
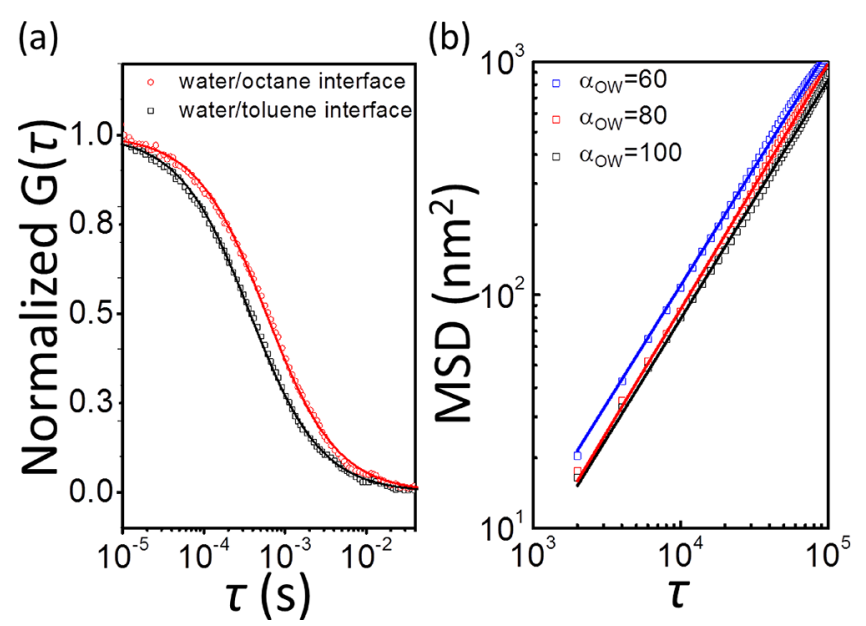

Figure 4. (a) Representative autocorrelation curves and their corresponding fits with eq 1 (solid lines) for JNPs at water/toluene (black squares) and water/octane (red circles) interfaces. (b) Simulated mean squared displacement versus lag time $\tau$ for ligandgrafted JNPs at interfaces with varying $\alpha_{\text {OW }}$ (proportional to interfacial tension).

DPD simulation showed a similar trend with decreasing the interfacial tension (Figure 4b). Taken together, these observations further indicated that polymer conformational change plays a dominant role in changing the $D_{\|}$of JNPs.

\section{CONCLUSIONS}

In summary, we used fluorescence correlation spectroscopy to measure and directly compare the translational diffusion of ligand-grafted JNPs and HNPs at water/oil interfaces. We found that the Janus character, originating from ligand asymmetry, can influence interfacial diffusion. $D_{\|}$of JNPs was smaller than $D_{\|}$of HNPs. Stepwise analysis using the DPD simulation revealed that these phenomena can be understood 
in the framework of normal Brownian motion. The polymeric ligands on a nanoparticle surface were deformed and oriented so that they occupy more surface area than the rigid particle, resulting in a larger effective radius than the nominal $R_{\mathrm{H}}$ obtained by measuring the diffusion in bulk solution. These results indicate that the Janus ligand arrangement not only strengthens adhesion to the interface as found previously but also affects interfacial diffusion.

\section{METHODS}

Janus and Homogeneous Nanoparticles. Thiol-terminated PEG, with a mean molecular weight of $5 \mathrm{~kg} / \mathrm{mol}$, was crystallized into lamellar single crystals that were able to adsorb native quantum dots. The side that had contact with the single crystals was grafted with PEG via the ligand exchange reaction. Next, 1-dodecanethiol was added to replace the oleate ligands on the quantum dots' surface that was not covered by the PEG. The surface of a resulting Janus nanoparticle was, therefore, compartmentalized by two portions that had ligands of opposite wettabilities. For comparison, chemically homogeneous nanoparticles were prepared by functionalizing quantum dots with thiol-terminated PEG on the whole surface via the ligand exchange reaction.

Sample Preparation for the FCS Studies. The source and purification of water and alkane are described in the Supporting Information. The water/n-alkane interfaces were prepared in an Attofluor cell chamber (Invitrogen, Leiden, Netherlands) equipped with a $150 \mu \mathrm{m}$ thick microscope cover glass slide as the bottom. An aluminum foil O-ring with an inner diameter of $0.5 \mathrm{~cm}$ and a thickness of approximately $0.3 \mathrm{~mm}$ was glued on the glass slide to restrict the sample volume for the aqueous phase. To prepare a water/ $n$-alkane interface suspended with nanoparticles, a defined amount of water was added into the cell. Then we added a nanoparticle dichloromethane solution on top of the aqueous solution. The dichloromethane was allowed to evaporate for typically $15 \mathrm{~s}$. Finally, alkane was added carefully on top of the water surface. The resulting surface coverage of tracer nanoparticles was around $1-2 \mu \mathrm{m}^{2}$ per particle.

Fluorescence Correlation Spectroscopy. FCS measurements were conducted on a commercial setup comprising the module ConfoCor 2 and an Axiovert 200 inverted microscope. The fluorescent particles were excited by an Ar laser $(488 \mathrm{~nm})$ focused by a water immersion microscope objective (C-Apochromat 40×, NA 1.2, Carl Zeiss, Germany). The fluorescent light was collected by the same objective, passed a LP530 long-pass emission filter and a confocal pinhole, and was finally directed to an avalanche photodiode detector that enabled single-photon counting. This arrangement resulted in the formation of a confocal detection volume $V_{\mathrm{d}}$ around the laser focus. The detection volume has a 3D Gaussian shape and typical dimensions of $\sim 200-250 \mathrm{~nm}$ in the radial direction and $1-1.5 \mu \mathrm{m}$ in the axial direction. Only the fluorescence originating from species within $V_{\mathrm{d}}$ can be delivered to and detected by the avalanche photodiode detector.

For each measurement, more than 20 independent autocorrelation curves were recorded on different days. The experiments were repeated at least three times on different days with freshly prepared samples, and the experimental uncertainty was less than $10 \%$.

Description of the DPD Method. We employed a computational DPD method to investigate the diffusion of nanoparticles. The DPD method was proven to be an effective mesoscopic simulation tool to study events occurring on millisecond time scales and micrometer length scales via tracking the motion of coarse-grained particles (composed of a group of atoms or molecules). The fundamental equation in the DPD method is the Newton's equation of motion. For a particle $i$, each DPD bead is subjected to three types of forces described as follows:

$$
m_{i} \frac{\mathrm{d} v_{i}}{\mathrm{~d} t}=\vec{F}_{i}=\sum_{j} \vec{F}_{i j}^{\mathrm{C}}+\vec{F}_{i j}^{\mathrm{R}}+\vec{F}_{i j}^{\mathrm{D}}
$$

where $\vec{F}_{i j}^{C}, \vec{F}_{i j}^{R}$, and $\vec{F}_{i j}^{D}$ are the conservative force, the pairwise random force, and the dissipative force, respectively. The force acting on a particle is summed over all interbead forces between particles $i$ and $j$. The conservative force is weakly repulsive and given by

$$
\vec{F}_{i j}^{\mathrm{C}}=-\nabla V\left(r_{i j}\right)=\left\{\begin{array}{cc}
\alpha_{i j}\left(1-\frac{r_{i j}}{r_{\mathrm{c}}}\right) \vec{e}_{i j}, & r_{i j} \leq r_{\mathrm{c}} \\
0, & r_{i j}>r_{c}
\end{array}\right.
$$

where $r_{i j}$ is the distance between particles $i$ and $j, r_{i j}=\left|\vec{r}_{i j}\right|=\left|\vec{r}_{i}-\vec{r}_{j}\right|, \vec{e}_{i j}$ $=\vec{r}_{i j} / r_{i j}$. Here, $\alpha_{i j}$ is a parameter to determine the magnitude of the repulsive force between particles $i$ and $j$, and $r_{c}$ is the cutoff distance. Random force $\left(\vec{F}_{i j}^{\mathrm{R}}\right)$ and dissipative force $\left(\vec{F}_{i j}^{\mathrm{D}}\right)$ are given by

$$
\begin{array}{rr}
\vec{F}_{i j}^{\mathrm{R}}=\sigma \omega^{\mathrm{R}}\left(r_{i j}\right) \xi_{i j} \vec{e}_{i j}, & r_{i j} \leq r_{\mathrm{c}} \\
\vec{F}_{i j}^{\mathrm{D}}=-\gamma \omega^{\mathrm{D}}\left(r_{i j}\right)\left(\vec{e}_{i j} \cdot \vec{v}_{i j}\right) \vec{e}_{i j}, & r_{i j} \leq r_{\mathrm{c}}
\end{array}
$$

where $\vec{v}_{i j}=\vec{v}_{i}-\vec{v}_{j}, \sigma$ is the noise parameter, $\gamma$ is the friction parameter, and $\zeta_{i j}$ is the random number based on the Gaussian distribution. Here $\omega^{\mathrm{R}}$ and $\omega^{\mathrm{D}}$ are $r_{i j}$-dependent weight functions, which are given by

$$
\omega^{\mathrm{D}}\left(r_{i j}\right)=\left[\omega^{\mathrm{R}}\left(r_{i j}\right)\right]^{2}=\left\{\begin{array}{cc}
\left(1-\frac{r_{i j}}{r_{c}}\right)^{2}, & r_{i j} \leq r_{\mathrm{c}} \\
0, & r_{i j}>r_{\mathrm{c}}
\end{array}\right.
$$

The temperature is controlled by a combination of dissipative and random forces. The noise parameter $\sigma$ and friction parameter $\gamma$ are connected to each other by the fluctuation-dissipation theorem in the following equation:

$$
\sigma^{2}=2 \gamma k_{\mathrm{B}} T
$$

where $T$ is the temperature and $k_{\mathrm{B}}$ is the Boltzmann constant.

The spring force $\left(\vec{F}_{i j}^{S}\right)$ between two bonded particles was given by

$$
\vec{F}_{i j}^{S}=-k\left(r_{i j}-r_{0}\right) \vec{e}_{i j}
$$

where $k$ is the spring constant and $r_{0}$ is the equilibrium bond distance.

\section{ASSOCIATED CONTENT}

\section{(I) Supporting Information}

The Supporting Information is available free of charge at https://pubs.acs.org/doi/10.1021/acsnano.0c03291.

Additional material information, procedure to rule out the effect of contamination; details of dissipative particle dynamics simulations; effect of interfacial tension on interfacial diffusion; spatial-dependent diffusion coefficient as a function of proximity to the liquid/liquid interface; contact line pinning of rough nanoparticles; Figures S6-S9 (PDF)

\section{AUTHOR INFORMATION}

\section{Corresponding Authors}

Dapeng Wang - State Key Laboratory of Polymer Physics and Chemistry, Changchun Institute of Applied Chemistry, Chinese Academy of Sciences, Changchun 130022, People's Republic of China; Max Planck Institute for Polymer Research, 55128

Mainz, Germany; Email: wdp@ciac.ac.cn

Zhao-Yan Sun - State Key Laboratory of Polymer Physics and Chemistry, Changchun Institute of Applied Chemistry, Chinese Academy of Sciences, Changchun 130022, People's Republic of China; ○orcid.org/0000-0002-6357-3039; Email: zysun@ ciac.ac.cn 
Kaloian Koynov - Max Planck Institute for Polymer Research, 55128 Mainz, Germany; 이이이.org/0000-0002-40628834; Email: koynov@mpip-mainz.mpg.de

\section{Authors}

You-Liang Zhu - State Key Laboratory of Polymer Physics and Chemistry, Changchun Institute of Applied Chemistry, Chinese Academy of Sciences, Changchun 130022, People's Republic of

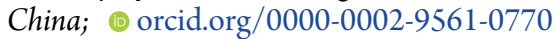

Yuehua Zhao - State Key Laboratory of Polymer Physics and Chemistry, Changchun Institute of Applied Chemistry, Chinese Academy of Sciences, Changchun 130022, People's Republic of China

Christopher Y. Li - Department of Materials Science and Engineering, Drexel University, Philadelphia, Pennsylvania 19104, United States; (1) orcid.org/0000-0003-2431-7099

Ashis Mukhopadhyay - Department of Physics, Wayne State University, Detroit, Michigan 48201, United States; (1) orcid.org/0000-0002-1366-5517

Hans-Jürgen Butt - Max Planck Institute for Polymer Research, 55128 Mainz, Germany; 이이.org/0000-0001-5391-2618

Complete contact information is available at:

https://pubs.acs.org/10.1021/acsnano.0c03291

\section{Author Contributions}

${ }^{\perp}$ D.W. and Y.Z. contributed equally to this work.

\section{Notes}

The authors declare no competing financial interest.

\section{ACKNOWLEDGMENTS}

This work was supported by the National Natural Science Foundation of China (Grants Nos. 21873094, 21833008, 21790344), International Science and Technology Cooperation Program of Jilin, China (Grant No. 20180414007GH), the Scientific Instrument Developing Project of the Chinese Academy of Sciences (Grant No. YJKYYQ20190084), and Key Research Program of Frontier Sciences, Chinese Academy of Sciences (Grants Nos. ZDBS-LY-SLH033 and QYZDY-SSWSLH027).

\section{REFERENCES}

(1) Bleibel, J.; Domínguez, A.; Oettel, M. Colloidal Particles at Fluid Interfaces: Effective Interactions, Dynamics and a Gravitation-Like Instability. Eur. Phys. J.: Spec. Top. 2013, 222, 3071-3087.

(2) Shi, S.; Russell, T. P. Nanoparticle Assembly at Liquid-Liquid Interfaces: From the Nanoscale to Mesoscale. Adv. Mater. 2018, 30, No. e1800714.

(3) Rezvantalab, H.; Shojaei-Zadeh, S. Capillary Interactions between Spherical Janus Particles at Liquid-Fluid Interfaces. Soft Matter 2013, 9, 3640-3650.

(4) Park, B. J.; Lee, D. Equilibrium Orientation of Nonspherical Janus Particles at Fluid-Fluid Interfaces. ACS Nano 2012, 6, 782-790.

(5) Rezvantalab, H.; Drazer, G.; Shojaei-Zadeh, S. Molecular Simulation of Translational and Rotational Diffusion of Janus Nanoparticles at Liquid Interfaces. J. Chem. Phys. 2015, 142, No. 014701.

(6) Binks, B. P.; Fletcher, P. D. I. Particles Adsorbed at the OilWater Interface: A Theoretical Comparison between Spheres of Uniform Wettability and "Janus" Particles. Langmuir 2001, 17, 47084710 .

(7) Glaser, N.; Adams, D. J.; Boker, A.; Krausch, G. Janus Particles at Liquid-Liquid Interfaces. Langmuir 2006, 22, 5227-5229.

(8) Cheung, D. L.; Bon, S. A. F. Stability of Janus Nanoparticles at Fluid Interfaces. Soft Matter 2009, 5, 3969-3976.
(9) Luu, X. C.; Yu, J.; Striolo, A. Nanoparticles Adsorbed at the Water/Oil Interface: Coverage and Composition Effects on Structure and Diffusion. Langmuir 2013, 29, 7221-7228.

(10) Fernandez-Rodriguez, M. A.; Song, Y.; Rodríguez-Valverde, M. A. N.; Chen, S.; Cabrerizo-Vilchez, M. A.; Hidalgo-Alvarez, R Comparison of the Interfacial Activity between Homogeneous and Janus Gold Nanoparticles by Pendant Drop Tensiometry. Langmuir 2014, 30, 1799-1804.

(11) Ruhland, T. M.; Groschel, A. H.; Ballard, N.; Skelhon, T. S.; Walther, A.; Muller, A. H. E.; Bon, S. A. F. Influence of Janus Particle Shape on Their Interfacial Behavior at Liquid-Liquid Interfaces. Langmuir 2013, 29, 1388-1394.

(12) Park, B. J.; Brugarolas, T.; Lee, D. Janus Particles at an OilWater Interface. Soft Matter 2011, 7, 6413-6417.

(13) Fernandez-Rodriguez, M. A.; Rodriguez-Valverde, M. A.; Cabrerizo-Vilchez, M. A.; Hidalgo-Alvarez, R. Surface Activity of Janus Particles Adsorbed at Fluid-Fluid Interfaces: Theoretical and Experimental Aspects. Adv. Colloid Interface Sci. 2016, 233, 240-254.

(14) Kaz, D. M.; McGorty, R.; Mani, M.; Brenner, M. P.; Manoharan, V. N. Physical Ageing of the Contact Line on Colloidal Particles at Liquid Interfaces. Nat. Mater. 2012, 11, 138-142.

(15) Wang, A.; Rogers, W. B.; Manoharan, V. N. Effects of ContactLine Pinning on the Adsorption of Nonspherical Colloids at Liquid Interfaces. Phys. Rev. Lett. 2017, 119, 108004.

(16) Wang, D.; Yordanov, S.; Paroor, H. M.; Mukhopadhyay, A.; Li, C. Y.; Butt, H. J.; Koynov, K. Probing Diffusion of Single Nanoparticles at Water-Oil Interfaces. Small 2011, 7, 3502-3507.

(17) Stocco, A.; Mokhtari, T.; Haseloff, G.; Erbe, A.; Sigel, R. Evanescent-Wave Dynamic Light Scattering at an Oil-Water Interface: Diffusion of Interface-Adsorbed Colloids. Phys. Rev. E 2011, 83, No. 011601.

(18) Du, K.; Liddle, J. A.; Berglund, A. J. Three-Dimensional RealTime Tracking of Nanoparticles at an Oil-Water Interface. Langmuir 2012, 28, 9181-9188.

(19) Boniello, G.; Blanc, C.; Fedorenko, D.; Medfai, M.; Mbarek, N. B.; In, M.; Gross, M.; Stocco, A.; Nobili, M. Brownian Diffusion of a Partially Wetted Colloid. Nat. Mater. 2015, 14, 908-911.

(20) Nelson, A.; Wang, D. P.; Koynov, K.; Isa, L. A Multiscale Approach to the Adsorption of Core-Shell Nanoparticles at Fluid Interfaces. Soft Matter 2015, 11, 118-129.

(21) Boniello, G.; Stocco, A.; Gross, M.; In, M.; Blanc, C.; Nobili, M. Translational Viscous Drags of an Ellipsoid Straddling an Interface between Two Fluids. Phys. Rev. E: Stat. Phys., Plasmas, Fluids, Relat. Interdiscip. Top. 2016, 94, No. 012602.

(22) Ortega, F.; Ritacco, H.; Rubio, R. G. Interfacial Microrheology: Particle Tracking and Related Techniques. Curr. Opin. Colloid Interface Sci. 2010, 15, 237-245.

(23) Wang, X.; In, M.; Blanc, C.; Wurger, A.; Nobili, M.; Stocco, A. Janus Colloids Actively Rotating on the Surface of Water. Langmuir 2017, 33, 13766-13773.

(24) Stocco, A.; Chollet, B.; Wang, X.; Blanc, C.; Nobili, M. Rotational Diffusion of Partially Wetted Colloids at Fluid Interfaces. J. Colloid Interface Sci. 2019, 542, 363-369.

(25) Zhong, Y.; Zhao, L.; Tyrlik, P. M.; Wang, G. Investigating Diffusing on Highly Curved Water-Oil Interface Using ThreeDimensional Single Particle Tracking. J. Phys. Chem. C 2017, 121, $8023-8032$

(26) Song, Y.; Luo, M.; Dai, L. L. Understanding Nanoparticle Diffusion and Exploring Interfacial Nanorheology Using Molecular Dynamics Simulations. Langmuir 2010, 26, 5-9.

(27) Koplik, J.; Maldarelli, C. Diffusivity and Hydrodynamic Drag of Nanoparticles at a Vapor-Liquid Interface. Phys. Rev. Fluids 2017, 2, No. 024303.

(28) Colosqui, C. E.; Morris, J. F.; Koplik, J. Colloidal Adsorption at Fluid Interfaces: Regime Crossover from Fast Relaxation to Physical Aging. Phys. Rev. Lett. 2013, 111, No. 028302.

(29) Razavi, S.; Kretzschmar, I.; Koplik, J.; Colosqui, C. E. Nanoparticles at Liquid Interfaces: Rotational Dynamics and Angular Locking. J. Chem. Phys. 2014, 140, No. 014904. 
(30) Wang, D. P.; Hu, R. F.; Skaug, M. J.; Schwartz, D. K. Temporally Anticorrelated Motion of Nanoparticles at a Liquid Interface. J. Phys. Chem. Lett. 2015, 6, 54-59.

(31) Megens, M.; Aizenberg, J. Like-Charged Particles at Liquid Interfaces. Nature 2003, 424, 1014-1014.

(32) Wang, B.; Li, B.; Zhao, B.; Li, C. Y. Amphiphilic Janus Gold Nanoparticles via Combining "Solid-State Grafting-To" and "Grafting-From" Methods. J. Am. Chem. Soc. 2008, 130, 11594-11595.

(33) Cristofolini, L.; Orsi, D.; Isa, L. Characterization of the Dynamics of Interfaces and of Interface-Dominated Systems via Spectroscopy and Microscopy Techniques. Curr. Opin. Colloid Interface Sci. 2018, 37, 13-32.

(34) Yang, J.; Zhao, J.; Han, C. C. Lateral Mobility of Single Chains at a Liquid Polymer Interface. Macromolecules 2008, 41, 7284-7286.

(35) Papadakis, C. M.; Košovan, P.; Richtering, W.; Wöll, D. Polymers in Focus: Fluorescence Correlation Spectroscopy. Colloid Polym. Sci. 2014, 292, 2399-2411.

(36) Koynov, K.; Butt, H.-J. Fluorescence Correlation Spectroscopy in Colloid and Interface Science. Curr. Opin. Colloid Interface Sci. 2012, 17, 377-387.

(37) Niu, Q.; Wang, D. Probing the Polymer Anomalous Dynamics at Solid/Liquid Interfaces at the Single-Molecule Level. Curr. Opin. Colloid Interface Sci. 2019, 39, 162-172.

(38) Wang, D.; Pevzner, L.; Li, C.; Peneva, K.; Li, C. Y.; Chan, D. Y.; Müllen, K.; Mezger, M.; Koynov, K.; Butt, H.-J. Layer with Reduced Viscosity at Water-Oil Interfaces Probed by Fluorescence Correlation Spectroscopy. Phys. Rev. E 2013, 87, No. 012403.

(39) Dörr, A.; Hardt, S.; Masoud, H.; Stone, H. A. Drag and Diffusion Coefficients of a Spherical Particle Attached to a Fluid-Fluid Interface. J. Fluid Mech. 2016, 790, 607-618.

(40) Isa, L.; Samudrala, N.; Dufresne, E. R. Adsorption of SubMicron Amphiphilic Dumbbells to Fluid Interfaces. Langmuir 2014, 30, 5057-5063.

(41) Schellenberger, F.; Papadopoulos, P.; Kappl, M.; Weber, S. A.; Vollmer, D.; Butt, H.-J. Detaching Microparticles from a Liquid Surface. Phys. Rev. Lett. 2018, 121, No. 048002.

(42) Du, K.; Glogowski, E.; Emrick, T.; Russell, T. P.; Dinsmore, A. D. Adsorption Energy of Nano- and Microparticles at Liquid-Liquid Interfaces. Langmuir 2010, 26, 12518-12522.

(43) Wang, D.; Hu, R.; Mabry, J. N.; Miao, B.; Wu, D. T.; Koynov, K.; Schwartz, D. K. Scaling of Polymer Dynamics at an Oil-Water Interface in Regimes Dominated by Viscous Drag and DesorptionMediated Flights. J. Am. Chem. Soc. 2015, 137, 12312-12320.

(44) Hua, X.; Bevan, M. A.; Frechette, J. Reversible Partitioning of Nanoparticles at an Oil-Water Interface. Langmuir 2016, 32, 1134111352 .

(45) Danov, K. D.; Dimova, R.; Pouligny, B. Viscous Drag of a Solid Sphere Straddling a Spherical or Flat Surface. Phys. Fluids 2000, 12, 2711-2722.

(46) Fischer, T. M.; Dhar, P.; Heinig, P. The Viscous Drag of Spheres and Filaments Moving in Membranes or Monolayers. J. Fluid Mech. 2006, 558, 451-475.

(47) Pozrikidis, C. Particle Motion near and inside an Interface. J. Fluid Mech. 2007, 575, 333.

(48) Blawzdziewicz, J.; Ekiel-Jezewska, M. L.; Wajnryb, E. Hydrodynamic Coupling of Spherical Particles to a Planar Fluid-Fluid Interface: Theoretical Analysis. J. Chem. Phys. 2010, 133, 114703.

(49) Dani, A.; Keiser, G.; Yeganeh, M.; Maldarelli, C. Hydrodynamics of Particles at an Oil-Water Interface. Langmuir 2015, 31, $13290-13302$.

(50) Blawzdziewicz, J.; Ekiel-Jezewska, M. L.; Wajnryb, E. Motion of a Spherical Particle near a Planar Fluid-Fluid Interface: The Effect of Surface Incompressibility. J. Chem. Phys. 2010, 133, 114702.

(51) Ma, X.; Chen, W.; Wang, Z.; Peng, Y.; Han, Y.; Tong, P. Test of the Universal Scaling Law of Diffusion in Colloidal Monolayers. Phys. Rev. Lett. 2013, 110, No. 078302.

(52) Zhu, Y. L.; Liu, H.; Li, Z. W.; Qian, H. J.; Milano, G.; Lu, Z. Y. GALAMOST: GPU-Accelerated Large-Scale Molecular Simulation Toolkit. J. Comput. Chem. 2013, 34, 2197-2211.
(53) Danov, K. D.; Kralchevsky, P. A.; Naydenov, B. N.; Brenn, G. Interactions between Particles with an Undulated Contact Line at a Fluid Interface: Capillary Multipoles of Arbitrary Order. J. Colloid Interface Sci. 2005, 287, 121-134.

(54) Colosqui, C. E.; Morris, J. F.; Koplik, J. Colloidal Adsorption at Fluid Interfaces: Regime Crossover from Fast Relaxation to Physical Aging. Phys. Rev. Lett. 2013, 111, No. 028302.

(55) Tay, K. A.; Bresme, F. Wetting Properties of Passivated Metal Nanocrystals at Liquid-Vapor Interfaces: A Computer Simulation Study. J. Am. Chem. Soc. 2006, 128, 14166-14175.

(56) Kim, J. U.; Matsen, M. W. Positioning Janus Nanoparticles in Block Copolymer Scaffolds. Phys. Rev. Lett. 2009, 102, No. 078303.

(57) Ranatunga, R. J. K. U.; Kalescky, R. J. B.; Chiu, C. C.; Nielsen, S. O. Molecular Dynamics Simulations of Surfactant Functionalized Nanoparticles in the Vicinity of an Oil/Water Interface. J. Phys. Chem. C 2010, 114, 12151-12157.

(58) Isa, L.; Amstad, E.; Schwenke, K.; Del Gado, E.; Ilg, P.; Kroger, M.; Reimhult, E. Adsorption of Core-Shell Nanoparticles at LiquidLiquid Interfaces. Soft Matter 2011, 7, 7663-7675.

(59) Li, Z.; Geisel, K.; Richtering, W.; Ngai, T. Poly (NIsopropylacrylamide) Microgels at the Oil-Water Interface: Adsorption Kinetics. Soft Matter 2013, 9, 9939-9946.

(60) Garbin, V.; Jenkins, I.; Sinno, T.; Crocker, J. C.; Stebe, K. J. Interactions and Stress Relaxation in Monolayers of Soft Nanoparticles at Fluid-Fluid Interfaces. Phys. Rev. Lett. 2015, 114, 108301.

(61) Geisel, K.; Rudov, A. A.; Potemkin, I. I.; Richtering, W. Hollow and Core-Shell Microgels at Oil-Water Interfaces: Spreading of Soft Particles Reduces the Compressibility of the Monolayer. Langmuir 2015, 31, 13145-13154.

(62) Vasudevan, S. A.; Rauh, A.; Kroger, M.; Karg, M.; Isa, L. Dynamics and Wetting Behavior of Core-Shell Soft Particles at a Fluid-Fluid Interface. Langmuir 2018, 34, 15370-15382.

(63) Schwenke, K.; Isa, L.; Cheung, D. L.; Del Gado, E. Conformations and Effective Interactions of Polymer-Coated Nanoparticles at Liquid Interfaces. Langmuir 2014, 30, 12578-12586.

(64) Lane, J. M. D.; Grest, G. S. Spontaneous Asymmetry of Coated Spherical Nanoparticles in Solution and at Liquid-Vapor Interfaces. Phys. Rev. Lett. 2010, 104, 235501.

(65) Camerin, F.; Fernandez-Rodriguez, M. A.; Rovigatti, L.; Antonopoulou, M.-N.; Gnan, N.; Ninarello, A.; Isa, L.; Zaccarelli, E. Microgels Adsorbed at Liquid-Liquid Interfaces: A Joint Numerical/ Experimental Study. ACS Nano 2019, 13, 4548-4559. 